\title{
Post-common envelope PN, fundamental or irrelevant?
}

\author{
Orsola De Marco ${ }^{1,2}$, T. Reichardt ${ }^{1,2}$, R. Iaconi ${ }^{1,2}$, T. Hillwig ${ }^{3}$, \\ G. H. Jacoby ${ }^{4}$, D. Keller ${ }^{5}$, R. G. Izzard ${ }^{6}$, J. Nordhaus ${ }^{7}$ and \\ E. G. Blackman ${ }^{8}$
}

\author{
${ }^{1}$ Department of Physics \& Astronomy, Macquarie University, Sydney, NSW 2109, Australia \\ email: orsola.demarco@mq.edu.au \\ ${ }^{2}$ Astronomy, Astrophysics and Astrophotonics Research Centre, Macquarie University \\ ${ }^{3}$ Department of Physics \& Astronomy, Valparaiso University, Indiana, USA \\ ${ }^{4}$ Lowell Observatory, Flagstaff, AZ, USA \\ ${ }^{5} \mathrm{KU}$ Leuven, Belgium \\ ${ }^{6}$ Institute of Astronomy, Cambridge University, UK \\ ${ }^{7}$ National Technical Institute for the Deaf, Rochester Institute of Technology, NY, USA \\ ${ }^{8}$ University of Rochester, NY, USA
}

\begin{abstract}
One in $5 \mathrm{PN}$ are ejected from common envelope binary interactions but Kepler results are already showing this proportion to be larger. Their properties, such as abundances can be starkly different from those of the general population, so they should be considered separately when using $\mathrm{PN}$ as chemical or population probes. Unfortunately post-common envelope PN cannot be discerned using only their morphologies, but this will change once we couple our new common envelope simulations with PN formation models.
\end{abstract}

Keywords. planetary nebulae: general, hydrodynamics, stars: AGB and post-AGB, binaries (including multiple): close, stars: evolution, stars: statistics

\section{The fraction and chemistry of post-common envelope PN}

At least one in 5 planetary nebulae $(\mathrm{PN})$ derives from a common envelope (CE) binary interaction, where the $\mathrm{PN}$ is the ejected $\mathrm{CE}$ and the inner close binary is the result of the companion's in-spiral into the progenitor AGB star (Ivanova et al. 2013). This fraction is a lower limit because we cannot detect photometric variabilities $\lesssim 0.1 \mathrm{mag}$, due primarily to poor sampling of ground-based observations. A search carried out with the Kepler Space Telescope resulted in the detection of 4 variables out of 5 objects with data (De Marco et al. 2015). Of the 4 variables, one is a double-degenerate binary central star, and one is likely to be a pole-on binary. With variability amplitudes of 0.0007 and $0.0005 \mathrm{mag}$, neither would have been detected from the ground.

A binary search using data from Kepler II (K2), campaigns 0, 2, 7 and 11 is underway. Campaigns 0, 2 and 7 include a grand total of 15 PN, two of which have periodic variability consistent with binarity. The variability amplitudes, 0.05 and 0.02 mag, respectively, would not have been easily detected from the ground. However, the sensitivity of K2 is up to 5 times worse than for Kepler and neither of the 2 binaries detected with Kepler would have been detected by K2. In addition, 11 of the $15 \mathrm{~K} 2$ targets analysed are compact $\mathrm{PN}$, while all the Kepler targets are extended: the variability detection threshold is therefore much worse for the bulk of the K2 targets. K2, Campaign 11 contains 139 viable $\mathrm{PN}$, of which 22 are extended. Hopefully those data will return better statistics. The Kepler data show that low amplitude post-CE PN central binaries exist, as predicted 


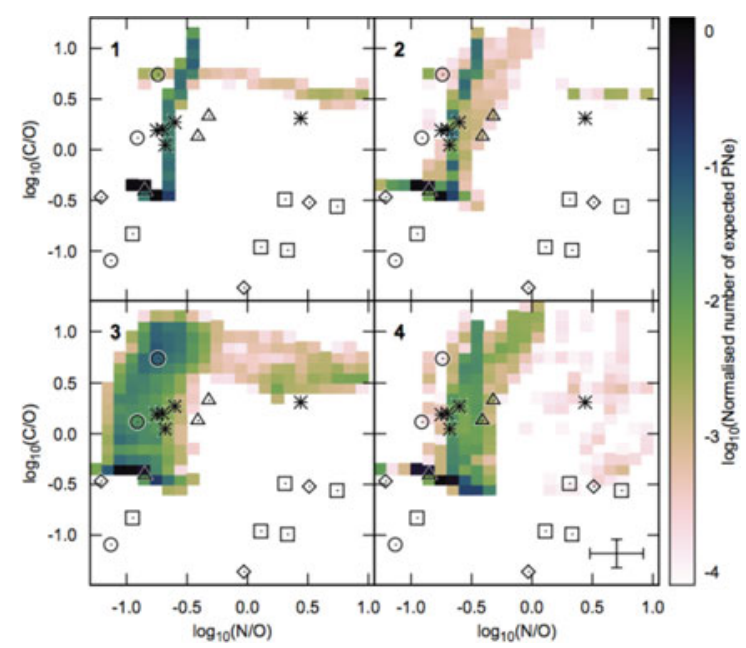

Figure 1. Observed vs. predicted $\mathrm{C} / \mathrm{O}$ and N/O ratios for $\mathrm{PN}$ produced via different channels: (1) single stars, (2) wind mass-loss from binary star origin, (3) CE merger + wind mass loss, (4) CE ejection. Observation symbols: round: round PN; star: elliptical PN; square: bipolar PN; triangular: elliptical PN with bipolar core, rhomboid: quadrupolar PN. Observations: Leisy \& Dennefeld (2006); Stanghellini et al. (2005). Figure from Keller, Izzard and Stanghellini, in prep.

and that the fraction of post-CE PN could be substantially larger than the lower limit of one in 5. This lower limit is already larger than the prediction from population synthesis of $9 \%$ for Solar metallicity of Izzard \& Keller (2015, Fig. 1, which are consistent with previous work, e.g., Yungelson et al. (1993); Nie et al. (2012); Madappatt et al. (2016)). An over-abundance of post-CE PN could be explained either if some single stars make under-luminous PN (Soker \& Subag 2005) or if some post-CE PN are mimics, such as for example Stroemgren spheres around hot stars (Frew \& Parker 2010).

Expectations of the properties of post-CE PN are different from those of PN from other evolutionary channels. Post-CE PN have a curbed AGB evolution, resulting in reduced $\mathrm{C} / \mathrm{O}$ ratios and lower s-process abundances. A secondary effect is that more massive stars are in binaries more often, something that could inflate the relative number of more massive stars in the post-CE PN population resulting in relatively larger $\mathrm{N} / \mathrm{O}$ ratios. In Fig. 2 we can compare the top-left and bottom-right panels where we see the relatively broader distributions in the post-CE channel and also the larger, though still small, proportion of $\mathrm{PN}$ at high $\mathrm{N} / \mathrm{O}$ and very low $\mathrm{C} / \mathrm{O}$ not present in the single star channel. The observations are still insufficient for a proper comparison, but it is already clear that the observed bipolar PN at low $\mathrm{C} / \mathrm{O}$ and mostly but not exclusively high N/O (more massive central stars) can only be (approximately) reproduced by the $\mathrm{CE}$ channel.

\section{Observed and simulated post-common envelope PN}

Unfortunately we cannot unambiguously recognise post-CE PN based on their morphology alone. Approximately 50 post-CE central stars are known to date and about two thirds of them are reasonably well imaged. As shown by Miszalski et al. (2009), many of these PN are approximately bipolar (e.g., A 41, K 1-2, Fig.2), but there is some diversity. Some PN display only a narrow waist (e.g., HaTr 4), sometimes with jets (A 63; jets not visible in Fig. 2). Ring nebulae (e.g., NGC 6337, Sp 1) are likely to be pole-on views of waists. More complex morphologies are also present: elliptical/bipolar PN with jets (NGC 6778, Fleming 1), more or less elliptical distributions of faint gas in which we see 


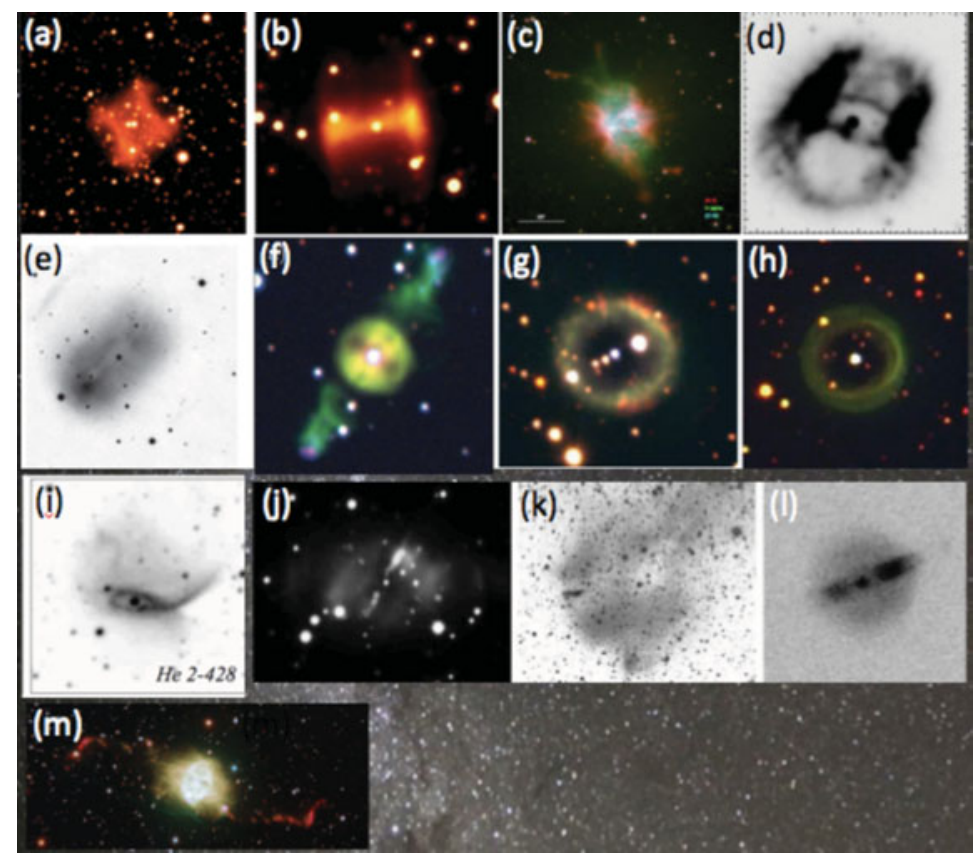

Figure 2. PN around post-CE central binaries; (a) A 63 (Mitchell et al. 2007); (b) HaTr 4 (Tyndall et al. 2012); (c) NGC 6778 (Guerrero \& Miranda 2012); (d) A 41 (Jones et al. 2010); (e) A 65 (Huckvale et al. 2013); (f) ETHOS 1 (Miszalski et al. 2011); (g) NGC 6337 (Hillwig et al. 2010); (h) Sp 1 (Hillwig et al. 2016); (i) He 2-428 (Santander-García et al. 2015); (j) K 1-2 (Corradi et al. 1999); (k) HFG 1 (De Marco 2009); (l) PN G135.9+55.9 (Napiwotzki et al. 2005); (m) Fleming 1 (Boffin et al. 2012).

superimposed stark and very collimated double (PN G 135.9; the similar structures in K 1-2 may be filaments (Miszalski et al. 2009)) or single jet-like structures (HFG 1).

The only way to understand how these shapes result from $\mathrm{CE}$ ejections is via simulations, such as those by García-Segura et al. (1999) or Huarte-Espinosa et al. (2012). Nobody to date has simulated a post-CE PN accounting for the common envelope ejection. Simulations of the CE interaction have shown that the ejection of the envelope takes place primarily on the orbital plane (Sandquist et al. 1998; Passy et al. 2012). We have recently carried out a 1-million particle SPH simulation with the code PHANTOM (Iaconi et al. 2017) and the parameters of Passy et al. (2012), but starting the simulation at the time of Roche lobe overflow, something that allows the distribution of ejected gas to be more realistic. In addition, with $\mathrm{SPH}$ the entire gas distribution at the end of the simulation $(\sim 15$ years after Roche lobe overflow) is known, albeit with varying resolution (from $0.3 \mathrm{R}_{\odot}$ near the binary to $80 \mathrm{R}_{\odot}$ near the outer parts of the distribution).

This simulation has not considered the fast wind, nor the ionising radiation. We could plausibly assume that the central star at the end of our simulation is hotter than $\sim 20,000 \mathrm{~K}$. If so, both ionising radiation and a fast tenuous wind would start blowing into the dense CE structure. While this has not been done yet in these simulations, using this image we can already make the following considerations. If a fast wind were to start at the end of the CE in-spiral it would meet with incredibly high densities, of the order of $10^{-8} \mathrm{~g} \mathrm{~cm}^{-3}$ in the vicinity of the stars (Fig. 3, left panel). Even the evacuated, funnel-like regions seen above and below the equatorial plane have high densities $\left(\sim 10^{-11} \mathrm{~g} \mathrm{~cm}^{-3}\right)$. By comparison typical PN simulations blow a fast wind into a much more tenuous medium with typical densities of $10^{-21} \mathrm{~g} \mathrm{~cm}^{-3}$, e.g., García-Segura (2006). 


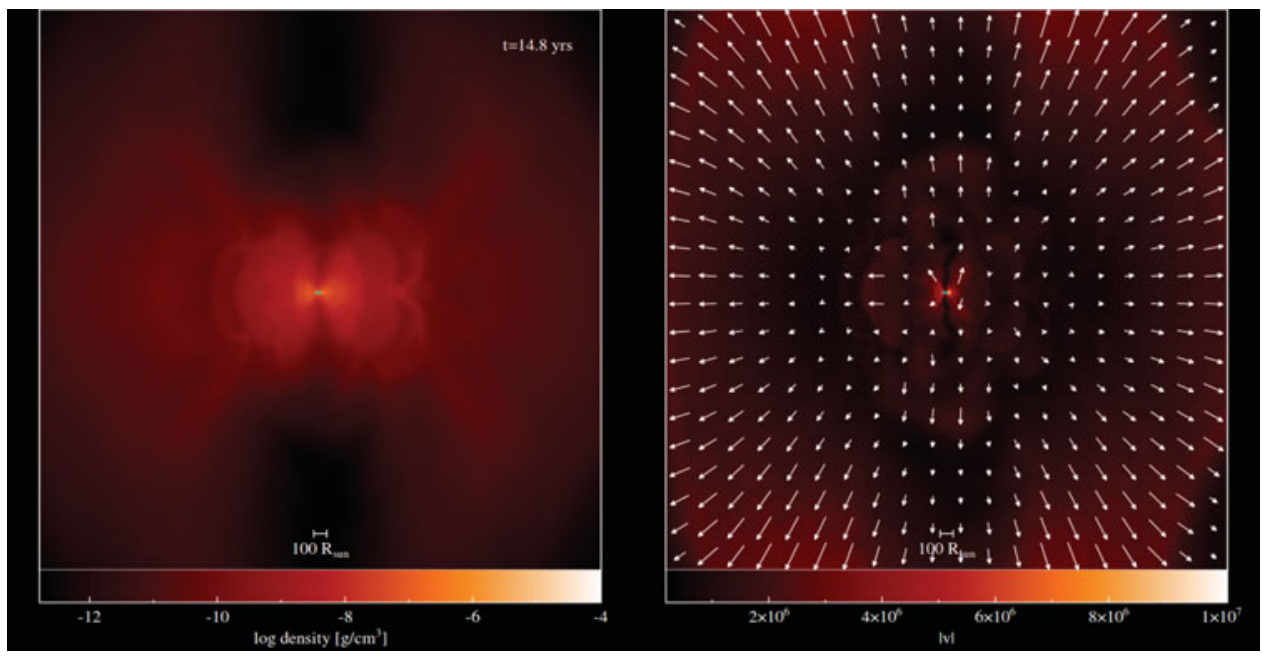

Figure 3. Density (left) and velocity (right, in $\mathrm{cm} \mathrm{s}^{-1}$; arrows indicate flow direction) slices perpendicular to the orbital plane at 14.8 years of an SPH CE simulation between a $0.88 \mathrm{M}_{\odot}$, RGB star and a $0.6 \mathrm{M}_{\odot}$ companion, carried out with the PHANTOM code (Iaconi et al. 2017).

This shows that the CE structure presents more or less a solid wall to the fast wind. On the other hand the entire CE is expanding at a range of speeds with the bulk of the volume expanding at $20 \mathrm{~km} \mathrm{~s}^{-1}$. Hence, in 100-1000 years the densities will decline by 5 to 8 orders of magnitude and the wind will start sweeping and penetrating the CE.

\section{References}

Boffin, H. M. J., Miszalski, B., Rauch, T., et al. 2012, Science, 338, 773

Corradi, R. L. M., Perinotto, M., Villaver, E. 1999, ApJ, 523, 721

De Marco, O. 2009, PASP, 121, 316

De Marco, O., Long, J., Jacoby, G. H., et al. 2015, MNRAS, 448, 3587

Frew, D. J. \& Parker, Q. A. 2010, PASA, 27, 129

García-Segura, G. 2006, in IAU Symp., 234, ed. Barlow \& Méndez, 297

García-Segura, G., Langer, N., Różyczka, M., \& Franco, J. 1999, ApJ, 517, 767

Guerrero, M. A. \& Miranda, L. F. 2012, A\&3A, 539, A47

Hillwig, T. C., Bond, H. E., Afşar, M., \& De Marco, O. 2010, AJ, 140, 319

Hillwig, T. C., Jones, D., De Marco, O., Bond, H. E., et al. 2016, ApJ, 832, 125

Huarte-Espinosa, M., Frank, A., Balick, B., et al. 2012, MNRAS, 424, 2055

Huckvale, L., Prouse, B., Jones, D., Lloyd, M., et al. 2013, MNRAS, 434, 1505

Iaconi, R., Reichardt, T., Staff, J., De Marco, O., et al. 2017, MNRAS, 464, 4028

Ivanova, N., Justham, S., Chen, X., De Marco, O., et al. 2013, A\&\&A Rev., 21, 59

Izzard, R. G. \& Keller, D. 2015, in ASP Conf. Ser., 497, ed. Kerschbaum, Wing, \& Hron, 161

Jones, D., Lloyd, M., Santander-García, M., et al. 2010, MNRAS, 408, 2312

Leisy, P. \& Dennefeld, M. 2006, A\& $A, 456,451$

Madappatt, N., De Marco, O., \& Villaver, E. 2016, MNRAS, 470, 317

Miszalski, B., Acker, A., Parker, Q. A., \& Moffat, A. F. J. 2009, A\& $A$, 505, 249

Miszalski, B., Corradi, R. L. M., Boffin, H. M. J., Jones, D., et al. 2011, MNRAS, 413, 1264

Mitchell, D. L., Pollacco, D., O’Brien, T. J., et al. 2007, MNRAS, 374, 1404

Napiwotzki, R., et al. 2005, in AIOP Conf. Ser., 804, ed. Szczerba, Stasinska, Gorny, 173

Nie, J. D., Wood, P. R., \& Nicholls, C. P. 2012, MNRAS, 423, 2764

Passy, J.-C., De Marco, O., Fryer, C. L., Herwig, F., et al. 2012, ApJ, 744, 52

Sandquist, E. L., Taam, R. E., Chen, X., Bodenheimer, P., \& Burkert, A. 1998, ApJ, 500, 909 
Santander-García, M., Rodríguez-Gil, P., Corradi, R. L. M., et al. 2015, Nature, 519, 63

Soker, N. \& Subag, E. 2005, AJ, 130, 2717

Stanghellini, L., Shaw, R. A., \& Gilmore, D. 2005, ApJ, 622, 294

Tyndall, A. A., Jones, D., Lloyd, M., O'Brien, T. J., \& Pollacco, D. 2012, MNRAS, 422, 1804

Yungelson, L. R., Tutukov, A. V., \& Livio, M. 1993, ApJ, 418, 794

\section{Discussion}

Q: We have many wonderful common-envelope ejection simulations in the meeting. What are they teaching us about the formation of bipolar lobes? How can we utilize observations of lobe shapes and kinematics to constrain CE models?

LEAL-FERREIRA: You showed the case of this source in which we are not sure what is going on; maybe binary, maybe pulsation... Can a triple system fit that observation?

DE MARCO: I am not sure how that would work. We tried to think of various binaryrelated phenomena but nothing really stood out as a clear winner.

CoRradi: Given the important implications on the binary fraction of the results from Kepler, can you comment if there may be some selection bias in the sample? Could there be a selection bias in the sample of post $\mathrm{CE} \mathrm{PN}$ in general?

DE MARCO: There should be no bias because the PN analyzed are all those found in the Kepler field. The only bias is the usual one affecting the entire PN sample (size, brightness, etc). In general we should consider whether any of the CE PN's and CE central stars' characteristics make them inherently easier to select out. 